\title{
Correlates of birth origin effects on the development of stereotypic behaviour in striped mice, Rhabdomys
}

\author{
Megan A. Jones ${ }^{\mathrm{a}, *}$, Georgia J. Mason ${ }^{\mathrm{a}, \mathrm{b}, 1}$, Neville Pillay ${ }^{\mathrm{a}}$ \\ ${ }^{a}$ School of Animal, Plant, and Environmental Sciences, University of the Witwatersrand \\ ${ }^{\mathrm{b}}$ Department of Animal and Poultry Sciences, University of Guelph
}

\section{A R T I C L E I N F O}

Article history:

Received 6 October 2010

Initial acceptance 16 November 2010

Final acceptance 15 April 2011

Available online 17 May 2011

MS. number: 10-00681R

\section{Keywords:}

animal welfare

birth origin

captive breeding

Rhabdomys

stereotypic behaviour

striped mouse

wild animal

\begin{abstract}
The behaviour and physiology of wild animals born in zoos, laboratories and breeding centres can differ substantially from that of their wild-caught (WC) conspecifics. For instance, captive-born (CB) animals are typically more prone to developing abnormal repetitive behaviours. In captive striped mice, Rhabdomys, we first confirmed that birth origin predicted the emergence of stereotypic behaviour (SB), with $\mathrm{CB}$ mice being most at risk. Second, to investigate correlates of this birth origin effect, we tested WC and $\mathrm{CB}$ striped mice in behavioural tasks to quantify fear/anxiety, activity and perseveration, and measured faecal corticosterone to assess physiological stress. WC mice proved more fearful and less active than $\mathrm{CB}$ animals, and had higher levels of faecal corticosterone metabolites. These effects, however, were unrelated to SB. WC mice were also less perseverative and more behaviourally flexible than $\mathrm{CB}$ animals, traits that covary with SB. Third, a retrospective analysis of laboratory records showed that SB incidence was significantly lower in adult-caught than juvenile-caught striped mice, with juvenile males being the most severely affected by early removal from the wild. In conclusion, our results indicate that adult, but not juvenile WC striped mice are typically protected against SB development in captivity, despite having poorer welfare than their CB conspecifics. They also reveal profound behavioural changes in CB mice, changes suggestive of altered forebrain function, a hypothesis now needing direct testing.
\end{abstract}

(c) 2011 The Association for the Study of Animal Behaviour. Published by Elsevier Ltd. All rights reserved.
Wild animals are often bred in research laboratories, zoos and conservation centres. Several recent studies show that captive-born (CB) animals may differ behaviourally and physiologically from their wild counterparts, a potential problem when the aim of captive breeding is to conserve or study wild phenotypes. Some observed differences are unsurprising: $C B$ animals may lack the experience to perform certain natural behaviour patterns competently (e.g. golden-lion tamarins, Leontopithecus rosalia: Kleiman et al. 1990; black-footed ferrets, Mustela nigripes: Biggins et al. 1999; bank voles, Clethrionomys glareolus: Mathews et al. 2005), and they are also typically less scared of humans than captive wildcaught (WC) conspecifics (e.g. black rhinoceros, Diceros bicornis: Carlstead et al. 1999; capybaras, Hydrochoerus hydrochaeris: Nogueira et al. 2004; starlings, Sturnis vulgaris: Feenders \& Bateson 2011). Probably as a consequence of their reduced fear, CB animals also generally appear to have better welfare in captivity: for

\footnotetext{
* Correspondence: M. A. Jones. School of Animal, Plant, and Environmental Sciences, University of the Witwatersrand, Private Bag 3, Wits, 2050, South Africa. E-mail address: megan.jones@icon.co.za (M.A. Jones).

1 G. J. Mason is based at the Department of Animal \& Poultry Science, University of Guelph, 50 Stone Road East, Guelph, Ontario N1G 2W, Canada.
}

example, compared with WC conspecifics, CB pigtailed macaques, Macaca nemestrina, show reduced mortality after a stressor (Ha et al. 2000). Other differences between WC and CB animals, however, are somewhat counterintuitive: compared with captive WC conspecifics, the offspring of $\mathrm{CB}$ mongoose lemurs, Lemur mongoz, have greater mortality (Perry et al. 1992); female CB white rhinoceroses, Ceratotherium simum simum, often fail to conceive (Swaisgood et al. 2006); and zoo-housed CB Asian elephants, Elephas maximus, are likely to die prematurely (Clubb et al. 2008). These examples indicate that birth origin can have dramatic effects, both positive and/or negative, on the phenotypes of captive animals.

Birth origin also has a striking influence on the development of highly repetitive stereotypic behaviours (SBs) such as pacing or body rocking. Although SBs afflict at least 10000 captive zoo animals worldwide (Mason et al. 2007), in eight of the 11 species studied to date they are rare or absent in WC individuals, and more common in conspecifics born in captivity (Mason 2006; Latham \& Mason 2008). The hypothesized causes of SBs are two-fold. First, SBs may arise from poor adjustment to impoverished captive conditions, resulting in the sustained elicitation of highly motivated, but ultimately thwarted (i.e. frustrated), natural behaviour patterns (hereafter 'source behaviours'; frustrated motivation 
hypothesis). For example, bar chewing in laboratory mice, Mus musculus, derives from repeated attempts to escape (Nevison et al. 1999), whereas stereotypic digging in gerbils, Meriones unguiculatus, is triggered by the lack of a species-typical tunnel-shaped entrance to a nesting chamber (Wiedenmayer 1997). Second, SBs can arise secondarily to changes in areas of the forebrain, especially the neural pathways between the cortex and the basal ganglia, responsible for behavioural flexibility and for the inhibition of inappropriate and unsuccessful responses (abnormal behavioural control hypothesis; e.g. Garner 2006; Lewis et al. 2006; Graybiel 2008; Latham \& Mason 2010). Thus in a number of species, a relationship has been found between individual levels of SB and increased 'perseveration' ('the continuation or recurrence of an...activity without the appropriate stimulus'; Sandson \& Albert 1987, page 1736) and reduced behavioural flexibility (e.g. Garner \& Mason 2002). Furthermore, treatments that induce SB (e.g. deprivation rearing or dosing with psychostimulants) correspondingly induce both perseveration and changes to these brain regions (e.g. Robbins et al. 1990; Lewis et al. 2006; Latham \& Mason 2008). The likely causes of low/absent SB are therefore low motivational frustration, leading to the lack of repetition of source behaviours, and/or a well-functioning, species-typical forebrain that permits the ready inhibition of inappropriate behaviours. Both of these have been suggested to explain why SB is rarer in complex, naturalistic captive environments than it is in small barren cages (e.g. Latham \& Mason 2010). However, a third reason has also been proposed to explain why some animals do not stereotype, even when they are kept in impoverished cages. Within these impoverished, SB-motivating environments, nonstereotypic individuals are generally atypically inactive (Meyer-Holzapfel 1968; Altman 1999), and also often seem to have poorer welfare than their stereotypic cagemates (Mason \& Latham 2004). This suggests that in adverse captive conditions, inactivity is an alternative response to $\mathrm{SB}$, perhaps because it represents hiding as a result of fear or excessive resting/sleeping secondary to 'apathy' (defined here as a lack of interest or motivation; Marin 1990).

How birth origin influences the development of SBs is unknown. However, the hypothesized reasons for low SB performance suggest that this birth origin-induced variation is mediated by experiencedependent changes in (1) forebrain structure and function (affecting abilities to inhibit inappropriate behaviours); (2) motivational systems (affecting the degree to which natural behaviours are frustrated and/or animals are fearful or apathetic); and/or (3) the extent of animals' fearfulness or apathy (affecting the level of hiding behaviour and inactivity). This gap in our knowledge reflects the type of previous investigation into $\mathrm{CB}-\mathrm{WC}$ differences in SB. Previous reports have been either serendipitous findings from studies in which the main focus was not SB, or have comprised findings from the retrospective analysis of multizoo data of animals spread over numerous sites (Mason 2006). As a result, none have allowed investigation into the precursors or correlates of the influence of birth origin on SB in a way that could shed light on underlying mechanisms. In this study, using the striped mouse Rhabdomys, we had the unique opportunity to compare WC and CB conspecifics kept as study subjects in the same laboratory. These small, diurnal murid rodents are abundant in many southern African habitats (Skinner \& Chimimba 2005); they are not endangered, they offer the typical advantages of a rodent species (e.g. small body size, successful reproduction in captivity and short generation times; Schradin \& Pillay 2003) and, because they are diurnal (Schradin 2006), are easy to observe and are not prone to sleep disruption when tested during the day. The striped mouse is a particularly good model for studying birth origin effects on SB as about half of all CB striped mice reared in standard cages develop locomotor SBs (Schwaibold \& Pillay 2001; Jones et al. 2008, 2010a, b). This incidence of SB is also similar to that reported in a number of zoo species (e.g. brown bears, Ursus arctos, 48\%; clouded leopards, Neofelis nebulosa, 49\%; reviewed in Mason et al. 2007), as is the proportion of time that stereotypic striped mice engage in SBs (about 50\% of active time in striped mice [Nel 2003] compared with $48 \%$ in lions, Leo panthera, and $52 \%$ in spectacled bears, Tremarctos ornatus [reviewed in Clubb \& Mason 2007]). The current study comprised three experiments in which we assessed whether WC striped mice are less stereotypic than $C B$ animals (experiment 1), explored correlates of birth origin and SBs as preliminary investigations into potential mechanisms (experiment 2 ), and analysed historical data to identify any constraints on the protective effects of being WC (experiment 3 ).

\section{GENERAL METHODS}

All wild striped mice were trapped using PVC live traps (290 $\times 60 \mathrm{~mm}$ and $70 \mathrm{~mm}$ high) which were covered with grass for insulation, and set for 4 consecutive days. Traps were baited with half a handful of a mixture of oats, raisins, salt, peanut butter and vegetable oil, and contained water-moistened cotton wool for hydration and dry cotton wool for bedding. Traps were checked both early morning and late afternoon, immediately after the peak activity of Rhabdomys, ensuring that caught individuals were unlikely to spend more than $2 \mathrm{~h}$ in the traps. No trap deaths were recorded. Following capture, individuals were transferred into holding cages $(200 \times 150 \mathrm{~mm}$ and $150 \mathrm{~mm}$ high, containing wood shavings for bedding, a handful of hay for nesting material, and provisioned with mouse cubes and water bottles), and then transported by road to the University of the Witwatersrand. Thereafter, adults were housed individually (except during breeding) as wild grassland striped mice are naturally solitary living (Schradin \& Pillay 2005), and the tendency to fight often precludes the caging of same-sex groups after weaning age (M. Jones, personal observations). Striped mice used in these studies were ultimately euthanized using either an overdose of an inhalant anaesthetic (Isoflurane or Halothane) or via carbon dioxide asphyxiation. Approval for all studies was provided by the University of the Witwatersrand's Animal Ethics Screening Committee.

\section{EXPERIMENT 1: WC VERSUS CB MICE}

In this first experiment, we compared the incidence of SB in a cohort of WC striped mice to a randomly chosen subsample of their first-generation $C B$ offspring. Because previous work in striped mice has suggested that SB is genetically based, and also may be selected for over generations in captivity (Jones et al. 2010b), we used only F1 CB individuals to maximize the genetic similarity between WC and CB animals (so allowing us to distinguish between environmental and genetic effects). We predicted that WC striped mice would be less likely to develop SBs than their CB offspring.

\section{Methods}

\section{Study subjects}

WC striped mice (males: $N=11$; females: $N=15$; all adults at the time of capture) were trapped in a grassland locality (Honeydew; Gauteng; 27 ${ }^{\circ} 5^{\prime} \mathrm{S}, 2^{\circ} 4^{\prime} \mathrm{E}$ ) between July 2006 and May 2007 as part of ongoing studies into SB in captive wild animals. The comparison group of $\mathrm{CB}$ striped mice was a randomly selected group of the F1 offspring bred from 15 different WC breeding pairs (males: $N=36$; females: $N=34$ ) for use in other behavioural studies. 
Housing and husbandry

Striped mice were housed in the Milner Park Animal Unit at the University of the Witwatersrand, under partially controlled environmental conditions (14:10 h light:dark, lights on at 0500 hours; $20-24{ }^{\circ} \mathrm{C} ; 30-60 \%$ relative humidity). All striped mice were kept singly in standard Labotec cages ( $300 \times 200 \mathrm{~mm}$ and $150 \mathrm{~mm}$ high) from the time of capture (WC) or from shortly after weaning (CB). The cages contained about $2 \mathrm{~cm}$ of wood shavings as bedding, a PVC tubing nestbox ( $10 \times 10 \mathrm{~cm}$ and $15 \mathrm{~cm}$ high, open at both ends) provisioned with hay and paper towel as nesting material, and one cardboard toilet roll. Mouse cubes and water were provided ad libitum. A small amount ( $\pm 3 \mathrm{~g}$ ) of seed mix (sunflower, millet) was sprinkled in the cage daily, together with fresh fruit and/or vegetables $( \pm 10 \mathrm{~g})$.

\section{Procedure}

The behaviour of the WC mice was assessed twice, 1 month and 5 months after capture, using direct behavioural observations. Each assessment covered 5 days, and involved one 15 min observation session per day. We defined SB as a repetitive behaviour comprising at least three successive repetitions (see Table 1 for definitions of the various forms of SBs observed; also Jones et al. 2010a). Striped mice consistently displaying SB (i.e. the behaviour was observed in at least three of the five observation sessions) were classed as stereotypic, whereas those displaying no SB were classed as nonstereotypic (see Jones et al. 2010a for a rationale of this dichotomous scoring method, which was the standard protocol in this laboratory). CB striped mice were similarly observed for SB over two 5-day periods, 1 month after being housed individually in Labotec cages (i.e. at 2 months of age; young adulthood), and again when 7 months old.

\section{Statistical analysis}

We examined the effect of birth origin on SB using a logistic regression with a binomial logit function (Statistica 8.0, Statsoft Inc., Tulsa, OK, U.S.A.). We included birth origin and sex as categorical predictors, and SB status (stereotypic or nonstereotypic) as the dependent variable. In this and subsequent analyses, all tests were two tailed, and differences were considered significant when $\alpha \leq 0.05$.

\section{Results}

All striped mice that showed SB in the first observation period also showed SB during the second observation session. Similarly, all nonstereotypic striped mice identified as such in the first observation period remained nonstereotypic when observed for a second time. SB was thus stable over the 5-month period of data collection, validating our scoring system. Birth origin was a significant predictor of the incidence of SB (Wald $\chi_{1}^{2}=11.633, P<0.001$ ), with WC striped mice showing substantially less SB (1/26; 4\% stereotypic) than their $\mathrm{CB}$ counterparts $(40 / 70 ; 57 \%)$. We found no effect of sex on the incidence of SB (Wald $\chi_{1}^{2}=0.221, P=0.638$ ).

\section{Discussion}

As predicted, first-generation CB striped mice were substantially more likely to be stereotypic than their WC counterparts, confirming that striped mice are a suitable model for studying the influence of birth origin on SB development. In addition, the observed consistency of SB over time (i.e. after 1 month and 5 months of individual housing in Labotec cages) in both CB and WC individuals indicates that the manifestation of SB during the first observation session was not simply a transient differential reaction to a recent change in housing conditions, but a stable trait. Next, in experiment 2, to explore the association between birth origin and SB, we studied a subsample of these $C B$ and WC subjects in more detail, investigating (1) group differences in behaviour and physiology and (2) individual correlates of SB performance.

\section{EXPERIMENT 2: BIRTH ORIGIN CORRELATES OF SB}

The suggested biological causes of low SB (see Introduction) indicate three main mechanisms that could underpin the diminished incidence of SB in WC striped mice. In turn, these potential mechanisms predict different correlates of the low SB of WC animals. Here, we therefore collected various cross-sectional behavioural and physiological data to investigate the correlates suggested by the following three hypotheses: WC animals seldom stereotype because (1) subjects maturing in the wild have more flexible behaviour and are less perseverative; (2) WC individuals adjust better to captivity than CB conspecifics and are less frustrated by its confines; and/or (3) WC individuals adjust poorly to captivity, but express this as inactivity.

Over the last decade, noninvasive behavioural paradigms have been used with good success to quantify behavioural repetition in stereotypic subjects, and in groups of animals subjected to SBenhancing treatments such as nonenriched housing (e.g. bank voles: Garner \& Mason 2002; songbirds: Garner et al. 2003; black bears, Ursus americanus, and sun bears, Ursus malayanus: Vickery \& Mason 2003; parrots: Garner et al. 2003; and deer mice, Peromyscus maniculatus: Tanimura et al. 2008). These methods have included 'gambling' (guessing) tasks, and reversal and extinction learning procedures. However, many of these procedures require subjects to undergo extensive operant training, to which striped mice are poorly suited: a large proportion of animals (especially nonstereotypic or WC individuals) fail to complete sufficient trials for initial learning to occur (N. Pillay, personal observations, unpublished data), and furthermore, human handling and contact may well be a differential stressor for WC and CB animals, thus acting as a confound. To quantify behavioural flexibility/perseveration, we therefore used the four-arm maze (e.g. Lalonde 2002), a behavioural test that does not depend on previous training. Analysis of arm entry sequence in a four-arm maze (or a T-maze) is typically measured by scoring spontaneous alternation behaviour (SAB; defined fully in Methods), a commonly used index of perseveration in assessing obsessive compulsive disorder (OCD)-like phenotypes

Table 1

Form and definition of stereotypic behaviours observed in striped mice

\begin{tabular}{|c|c|}
\hline Form of stereotypic behaviour & Definition \\
\hline Cage climbing & Climbing on the cage lid, using either forelimbs or fore- and hindlimbs \\
\hline Circuit running & Running in the cage along a fixed route (frequently in a figure-of-eight pattern) \\
\hline Jack hammering & Jumping up and down on the hindlimbs, usually in a corner of the cage or, if a nestbox is present, on and off the nestbox \\
\hline Prelooping & $\begin{array}{l}\text { Climbing upside down on the cage lid with forelimbs and then hindlimbs, moving backwards along lid, then dropping } \\
\text { down by releasing the hindlimbs first }\end{array}$ \\
\hline Somersaulting & Backward flipping, with or without touching the cage lid \\
\hline Windscreen wiping & $\begin{array}{l}\text { With hindpaws stationary or moving only slightly on the cage floor, the forelimbs oscillate to-and-fro in a large arc } \\
\text { against the cage walls }\end{array}$ \\
\hline
\end{tabular}


(e.g. Yadin et al. 1991; Ulloa et al. 2004) that are characterized by perseverative deficits in inhibitory control (Joel et al. 2008). SAB is also sensitive to the effects of lesions in corticostriatal circuitry (Divac et al. 1975; Delatour \& Gisquet-Verrier 1996) and to the administration of drugs (e.g. Quinpirole) that are standard pharmacological models of OCD in rodents (Joel et al. 2008). In addition to scoring $\mathrm{SAB}$, we used sequential analysis (SA) to assess the pattern of arm entries in the maze because, although $S A B$ detects whether individuals perseverate in their arm choice rather than alternating among the different arms, it does not assess whether an animal predictably enters a particular arm in a regularly repeated sequence.

We made use of a second behavioural test, the light-dark box, to assess fear/anxiety. Here, the proportion of time spent in the dark compartment and the latency to emerge from the dark compartment after first entry into it are commonly used indexes of rodent anxiety (Belzung \& Griebel 2001; Bourin \& Hascoët 2003), correlating closely with scores from other behavioural measures of anxiety (e.g. open field, Kim et al. 2002). Pharmacological studies show that the tendency to prefer the dark compartment and the latency to emerge from it are reduced by anxiolytic drugs and potentiated by anxiogenic drugs (Imaizumi et al. 1994). We also collected faecal samples to measure circulating levels of the stress hormone corticosterone while striped mice were in their home cages. Faecal corticosterone levels are an accepted measure of hypothalamic - pituitary-adrenal (HPA) axis activity, with high levels often reflecting increased stress/fear (Touma \& Palme 2005). Faecal sampling has fewer welfare concerns than blood sampling because it is noninvasive (Touma et al. 2003) and, in striped mice, faecal pellets are easier to collect than urine samples. Faecal sampling also gives a picture of the levels of free, unbound hormone over a period of several hours (e.g. Touma et al. 2003, Touma \& Palme 2005). Finally, we assessed levels of activity in the home cage, as well as in the four-arm maze (centre crossings; e.g. Hliňák \& Krejči 2006). Previous studies have reported a link between hyperactivity and SB in bank voles (Garner \& Mason 2002), while hypoactivity can potentially reflect hiding behaviour and/or apathy (Meyer-Holzapfel 1968; Broom \& Johnson 1993).

We made three predictions. (1) If forebrain function were altered in $\mathrm{CB}$ individuals in the same way as in deprivation-reared primates and nonenriched-reared deer mice (see Introduction), we would predict (1a) higher SAB scores and (1b) lower SA scores in WC compared with $C B$ animals (both measures reflecting lower perseverative tendencies). Furthermore, if reduced perseveration and low SB were to share a common underlying mechanism, we would predict higher SAB scores and lower SA scores in nonstereotypic striped mice, for these traits to covary, and for any birth origin effect on SA and SAB to vanish if SB were controlled for in the model. (2) If WC striped mice were better adjusted to captivity than CB animals, we would expect lower levels of fear/anxiety and hiding: individuals would (2a) spend more time in the light compartment of the light-dark box, (2b) show shorter latencies to re-enter the light compartment after their first entry into the dark compartment, (2c) have reduced corticosteroid levels and (2d) spend less time in their nestboxes during peak activity times. Furthermore, if these effects were related to the lower incidence of SB in WC mice, we would expect reduced fear/anxiety in all nonstereotypic individuals. (3) In contrast, if aversive emotional states were suppressing SB by inducing inactivity, we would expect WC animals to be more fearful/anxious and less active (corollaries of predictions $2 \mathrm{a}-\mathrm{c}$ above) and, again, if such responses were related to the low SB of WC mice, we would also predict nonstereotypic animals in general to have higher anxiety, lower activity and to spend more time hiding/being inactive. Similar to prediction (1), if either predictions (2) or (3) were correct, we would also expect that any birth origin effects on these variables would disappear if we included SB as a categorical predictor in the statistical model.

\section{Methods}

\section{Study subjects and housing}

First, to assess group differences in behaviour and physiology between WC and CB striped mice, we used all 26 WC striped mice from experiment 1, and a random subsample of 14 of their F1 CB offspring (eight stereotypic, six nonstereotypic; data set 1 ). This data set, however, did not allow us to tease apart the relative correlates of birth origin and SB because only one WC mouse was stereotypic. We therefore created a second data set, for which we generated a larger sample of WC individuals that included all striped mice trapped subsequent to the first cohort and that developed SB $(N=4)$. This data set, 2 , thus comprised five stereotypic and 25 nonstereotypic WC animals. Sample sizes were ultimately unbalanced as, while we sought to use all available animals, we were also constrained by the number of individuals that were available to keep for prolonged periods in captivity and/or not being used in other projects, as well as the number of suitable WC animals trapped after experiment 1.

We collected all data when WC mice were about 15 months old (based on their body size, we assumed that WC individuals were on average 3 months old when captured), and when the age of $C B$ mice ranged between 13 and 16 months. Housing conditions were identical to those described in experiment 1.

\section{Procedure}

Behavioural data were collected from testing in two apparatuses (four-arm maze and light-dark box) and from home cage observations. Test sessions took place between 0700 and 1100 hours, during which time Rhabdomys is most active (Pillay 2000). A minimum of $24 \mathrm{~h}$ was allowed between sessions. All apparatuses were illuminated from above with fluorescent lighting, and the sessions were recorded by a video camera positioned directly above the apparatuses or to the side of the home cage. The apparatuses were cleaned between tests, using soap and water. Video recordings were scored using Observer 5.0 (Noldus Information Technology, Wageningen, The Netherlands).

Four-arm maze. The four-arm maze consisted of four enclosed arms ( $7.5 \times 7.5 \mathrm{~cm}$ and $15 \mathrm{~cm}$ high), constructed from clear PVC, connected to a central area $(10 \times 10 \mathrm{~cm}$ and $20 \mathrm{~cm}$ high $)$. A subject was placed into the central area, and its behaviour recorded for $10 \mathrm{~min}$. The frequency of arm entries was recorded as a measure of locomotor activity. We followed the methods of Hliňák \& Krejči (2006) to calculate spontaneous alternation scores, defining an SAB score for a series of four-arm entries (a tetrad) as the ratio of actual arms entered to the possible number of arms that could have been entered. Thus, in the arm entry sequence $12342 . .234$, an alternation score for each tetrad was calculated as follows: for the first tetrad (12342...234), the mouse entered four different arms out of a possible four, giving an alternation score of $4 / 4=1$; for the second tetrad (12342...234), it entered three arms out of a possible four, and hence scored $3 / 4=0.75$, with the last three entries of the sequence $(12342 \ldots 234)$ not being considered because these did not constitute a complete tetrad. Total SAB scores for the trial were calculated by averaging $\mathrm{SAB}$ scores across all tetrads in a sequence, with low overall scores representing a tendency to enter a more restricted number of arms and to make more repeat visits of the same arm.

We used sequential analysis to assess the predictability of a striped mouse entering a particular arm following entry into another particular arm. For example, a striped mouse might have 
the sequence 1234123412341234 , repeating the same pattern of arm entries, yet would be judged, using spontaneous alternation, as not perseverating (all tetrads would receive a score of 1 ). Sequential analysis, however, can detect this form of perseveration by assessing whether one behavioural element is more or less likely than chance to follow another behavioural element (see Van Hooff 1982). For each individual, the sequence of arm entries was coded into transition matrices with the current behavioural element (an entry into one of the arms) represented in the columns and the preceding arm entered represented in the rows. Using the software Matman (Noldus Information Technology), we calculated the adjusted residuals (i.e. differences between observed and expected values for each transition frequency) for each matrix and then used the generated $\chi^{2}$ value for each matrix as an index of routine formation (the higher the $\chi^{2}$ value, the more predictable a mouse's pattern of arm entry; thus unlike the SAB score, here high scores mean more predictable). One individual (WC, nonstereotypic female) was excluded from SAB and SA analysis because she remained in one arm for the entire trial, precluding score calculation.

Light-dark box. The light-dark box comprised a glass tank divided in half (each half $30 \times 22.5 \mathrm{~cm}$ and $30 \mathrm{~cm}$ high with three ventilation holes in the Perspex lid); the two halves were connected by a $6 \times 6 \mathrm{~cm}$ opening on the floor of the tank. One half of the tank, the dark compartment, had black walls and lid; the other half, the light compartment, had clear walls and lid. A subject was placed in the light compartment facing away from the opening into the dark compartment, and its behaviour recorded for $5 \mathrm{~min}$. We scored the proportion of time in the dark compartment and the latency to return to the light compartment after first entry into the dark compartment.

Home cage observations. Subjects were videorecorded in their home cage for $40 \mathrm{~min}$ per day for 3 consecutive days. From the recordings, we observed the behaviour of subjects over two $10 \mathrm{~min}$ periods (at minutes 5-15 and 25-35), scoring the following behaviours: time spent in the nest (inside the nestbox, usually inactive, resting or sleeping); time spent outside the nest (activity outside the nestbox, excluding SB); and also total duration of SB (SB being defined in experiment 1 ). We did not consider inactivity outside the nestbox because this was very rarely seen. We also assessed the forms of SB shown by WC and CB striped mice since previous work in passerine birds has shown this to differ between CB and WC individuals (Keiper 1969).

Faecal corticosterone metabolites. Individual faecal samples were collected between 0800 and 1000 hours (timing of collection was standardized to minimize variation caused by fluctuation in circadian corticosterone production; Touma \& Palme 2005). To collect a sample, a striped mouse was transferred from its home cage into a clean plastic container where it remained until five faecal pellets had been collected or $1 \mathrm{~h}$ had elapsed, whichever occurred sooner. The sampling period was time limited to avoid the stress of the procedure contaminating the sample (e.g. Touma et al. 2003, 2004; Latham \& Mason 2010); rodent faecal samples usually represent plasma levels of unbound hormone at least $4 \mathrm{~h}$ earlier (Touma et al. 2003). The procedure was repeated the following week if insufficient pellets were collected. Samples were frozen immediately after collection, and later sent for analysis to Gordon Laboratories (Sedgefield, Stockton-on-Tees, U.K.). In total, samples were analysed for all 14 CB mice (eight stereotypic, six nonstereotypic) and 17 WC mice (five stereotypic, 12 nonstereotypic; samples were unfortunately not collected from the remaining $13 \mathrm{WC}$ individuals since these animals had been euthanized prior to the approval of this part of the experiment).

\section{Data analysis}

Data were analysed in general linear models (GLMs) or generalized linear models (GLZ). For data set 1 , we compared group differences in behaviour and physiology using logistic models with birth origin and sex as categorical predictors. To investigate individual correlates of SB (data set 2), we similarly used logistic models, but this time included birth origin, sex and SB status as the categorical predictors. For the quantitative SB data on home cage time budgets, further analyses were run to compare how time consuming SBs were in WC and CB striped mice (we included birth origin as the categorical predictor and how time-consuming SBs were in stereotypic striped mice as the dependent variable; nonstereotypic individuals were excluded) and to assess the relationship between our two measures of perseveration (SAB and SA) and how time consuming SBs were.

\section{Results}

\section{Birth origin effects on behaviour and physiology}

Summary data and results from statistical analyses of the two groups, WC and CB, in data set 1 are given in Table 2. Overall, CB striped mice were more perseverative than WC individuals: in the four-arm maze, birth origin was a significant predictor of SA scores, with $\mathrm{CB}$ individuals entering the arms in a more predictable sequence than WC animals (thus having higher scores). CB striped mice also showed a strong trend towards having lower SAB scores, indicating more repeated entries into a limited number of maze arms. We found no effect of sex on either SA or SAB scores.

Compared with $\mathrm{CB}$ striped mice, WC individuals spent more of the trial in the dark compartment of the light-dark box, and took longer to emerge from the dark compartment after first entry into it. Sex did not predict the proportion of time spent in the dark or of the latency to emerge therefrom. Corticosterone metabolite levels

Table 2

Behavioural and physiological results and analyses for CB and WC striped mice from data set 1

\begin{tabular}{|c|c|c|c|c|}
\hline & \multicolumn{2}{|c|}{ Results: median value (1st and 3rd quartiles) } & \multicolumn{2}{|c|}{ Analyses: Wald $\chi_{1}^{2}(P)$} \\
\hline & $\mathrm{CB}(N=14)$ & WC $(N=26)$ & Birth origin & Sex \\
\hline SAB score & $0.83(0.79,0.86)$ & $0.87(0.84,0.88)$ & $3.485(0.062)$ & $0.095(0.758)$ \\
\hline SA chi-square & $25.00(19.30,29.90)$ & $18.50(16.30,22.10)$ & $8.469(0.004)$ & $0.738(0.390)$ \\
\hline Time in dark compartment (\%) & $47.70(39.56,52.67)$ & $64.62(52.21,75.31)$ & $13.840(<0.001)$ & $0.330(0.565)$ \\
\hline Latency to emerge (s) & $14.78(8.35,21.81)$ & $36.03(24.53,62.51)$ & $377.986(<0.001)$ & $0.027(0.869)$ \\
\hline Faecal corticosterone $(\mu \mathrm{g} / \mathrm{ml})$ & $7.52(6.76,8.08)$ & $8.51(7.95,9.82)$ & $5.480(0.019)$ & $3.710(0.054)$ \\
\hline Frequency of arm entries & $75.50(59.50,111.75)$ & $45.50(32.00,66.75)$ & $7.892(0.005)$ & $0.050(0.823)$ \\
\hline Time in nestbox (\%) & $67.62(35.62,83.63)$ & $98.40(83.83,100.00)$ & $7.434(0.006)$ & $1.380(0.240)$ \\
\hline Home cage activity (\%) & $32.39(16.37,64.38)$ & $1.56(0.00,16.16)$ & $7.434(0.006)$ & $0.349(0.560)$ \\
\hline
\end{tabular}


were significantly higher in WC than $\mathrm{CB}$ mice, and tended to be higher in females than in males. In the home cage, WC striped mice, irrespective of sex, spent more time in the nestbox. In both the home cage and the four-arm maze, WC individuals were also less active than $\mathrm{CB}$ individuals. Neither of the activity measures was influenced by sex, but time in the nest was significantly predicted by the proportion of time in the dark compartment of the light-dark box.

\section{Individual correlates of $S B$}

In the analyses of data set 2 , we tested whether these betweengroup differences in perseveration, anxiety/fear and home cage activity were related to individual susceptibilities to stereotype. When we examined the concurrent effects of birth origin and stereotypy status on perseveration, the main effect of birth origin disappeared, and stereotypic status was a strong predictor of both SA (Wald $\chi_{1}^{2}=9.45, P=0.002$ ) and SAB scores (Wald $\chi_{1}^{2}=10.99$, $P<0.001$; Fig. 1a, b). In both cases, stereotypic individuals were more perseverative than nonstereotypic animals. The analysis of SA scores also revealed a significant interaction of birth origin and stereotypic status (Wald $\chi_{1}^{2}=4.25, P=0.039$ ): while stereotypic mice always showed more predictable sequences of arm entry, this effect was particularly pronounced in WC stereotypic mice.

When quantitative SB time budget data were analysed (nonstereotypic striped mice being excluded from these analyses), birth origin was a significant predictor of the severity of SBs (Wald $\left.\chi_{1}^{2}=8.01, P=0.005\right)$ : the few stereotypic WC mice actually had more time-consuming SBs than WC conspecifics, spending around 76.32\% (45.40, 77.26\%; median, 1st and 3rd quartiles) of their time engaged in SB, compared with $23.93 \%$ (13.05, 31.51\%) of stereotypic CB striped mice. SB forms observed in WC striped mice were also less diverse than in $\mathrm{CB}$ animals: all five WC individuals were circuit-
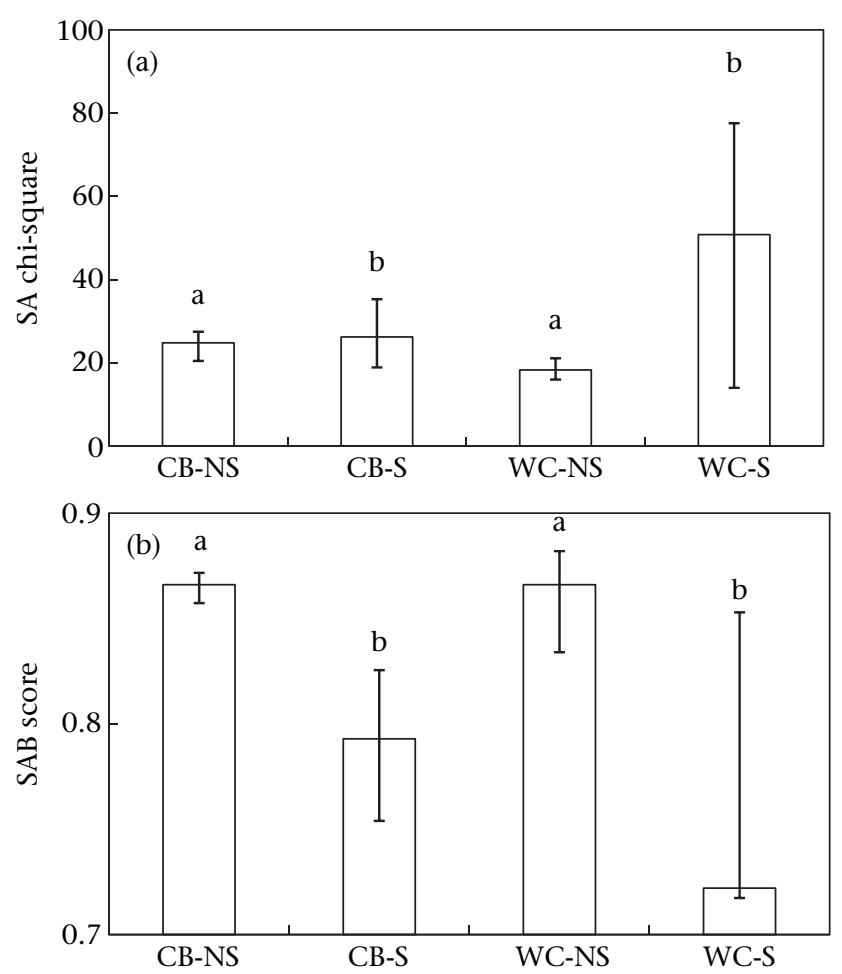

Figure 1. Perseveration in the four-arm maze. (a) Sequential analysis chi-square. (b) Spontaneous alternation score. $\mathrm{CB}=$ captive-born; $\mathrm{WC}=$ wild-caught; NS = nonstereotypic; $\mathrm{S}=$ stereotypic. The same alphabet letters denote groups that are not significantly different. Bars = median; whiskers $=1$ st and 3rd quartiles. runners, whereas stereotypic $\mathrm{CB}$ striped mice showed a greater intra- and interindividual diversity of SBs such as windscreen wiping and prelooping in addition to circuit running. The time spent stereotyping, like the incidence of $\mathrm{SB}$, was predicted by SA scores (Wald $\chi_{1}^{2}=3.918, P=0.048$ ) but not, however, by SAB (Wald $\left.\chi_{1}^{2}=1.531, P=0.216\right)$. That is, the striped mice that had the most time-consuming SBs also had the highest SA scores, thus showing greater perseveration in the four-arm maze.

For measures of fear/anxiety, however, the main effect of birth origin persisted in data set 2 and with SB status as a categorical predictor: WC individuals thus spent a greater proportion of the trial in the dark compartment (Wald $\chi_{1}^{2}=7.88, P=0.005$; Fig. 2a), took longer to emerge from the dark compartment after first entry into it (Wald $\chi_{1}^{2}=10.71, P=0.001$; Fig. $2 \mathrm{~b}$ ) and, regardless of stereotypy status, had higher levels of faecal corticosterone metabolites (Wald $\chi_{1}^{2}=4.78, P=0.029$; Fig. 3). Correspondingly, the time devoted to SB in stereotypic mice was also unrelated to these measures of anxiety/stress.

In contrast, activity level in the four-arm maze was significantly predicted by SB status in analyses of data set 2, with stereotypic animals, irrespective of birth origin, having a higher frequency of arm entries than nonstereotypic striped mice (Wald $\chi_{1}^{2}=9.12$, $P=0.003$; Fig. 4 a). Home cage activity was also similarly related to stereotypy status: both CB and WC stereotypic striped mice spent more time active than their nonstereotypic counterparts (Wald $\chi_{1}^{2}=18.00, P<0.001$; Fig. 4 b). In terms of nestbox use, stereotypic WC striped mice also spent the least amount of time in the nestbox, followed by stereotypic CB striped mice, nonstereotypic CB striped mice and, finally, nonstereotypic WC mice (birth origin*stereotypy status: Wald $\chi_{1}^{2}=7.73, P=0.005$; Fig. 4 c).
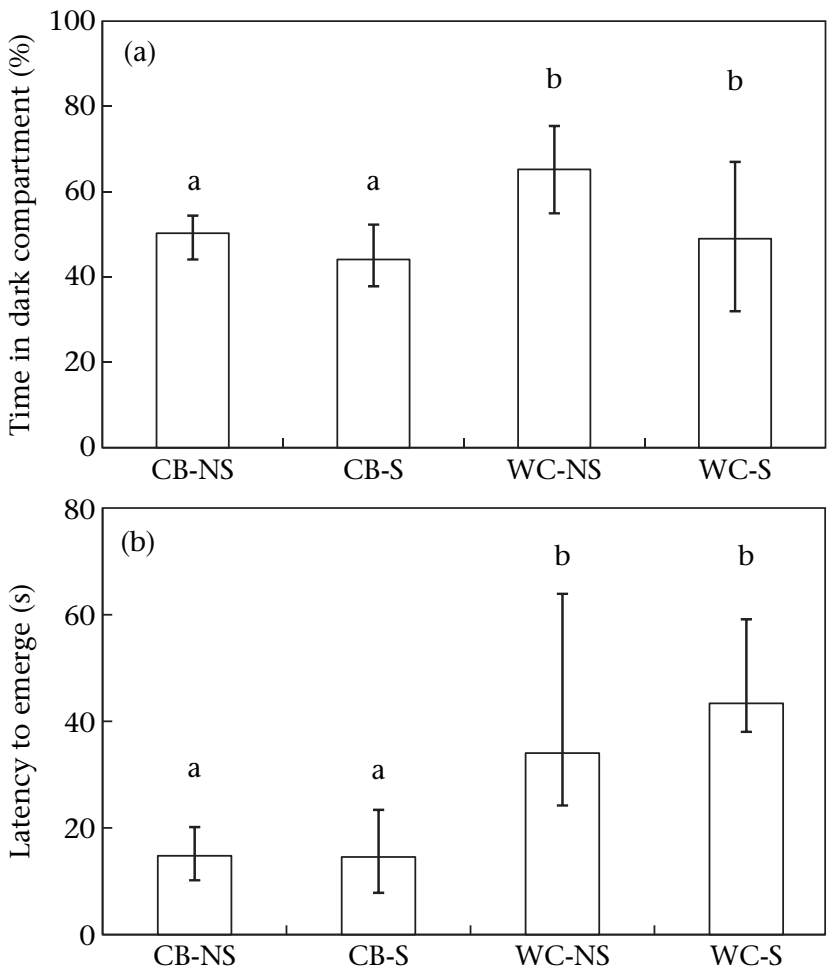

Figure 2. Anxiety/fearfulness in the light-dark box. (a) The percentage of time spent in the dark compartment. (b) Latency to first emergence from the dark compartment. $\mathrm{CB}=$ captive-born; $\mathrm{WC}=$ wild-caught; $\mathrm{NS}=$ nonstereotypic; $\mathrm{S}=$ stereotypic. The same alphabet letters denote groups that are not significantly different. Bars = median; whiskers $=1$ st and 3rd quartiles. 


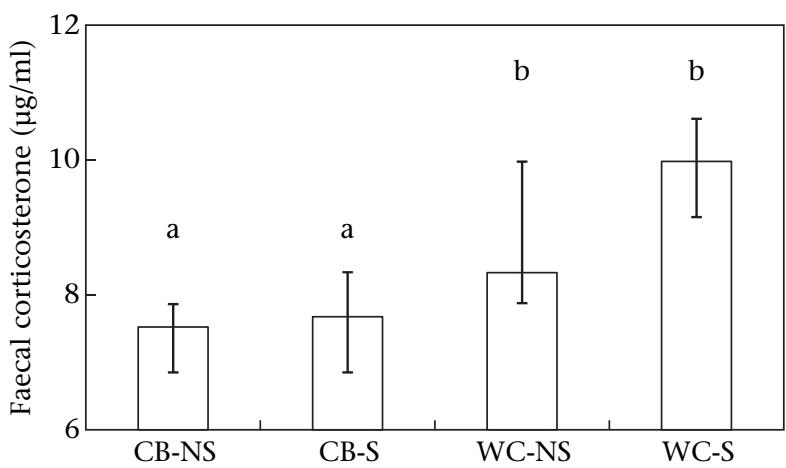

Figure 3. Faecal corticosterone. $\mathrm{CB}=$ captive-born; $\mathrm{WC}=$ wild-caught; $\mathrm{NS}=$ nonstereotypic; $\mathrm{S}=$ stereotypic. The same alphabet letters denote groups that are not significantly different. Bars $=$ median; whiskers $=1$ st and 3rd quartiles.
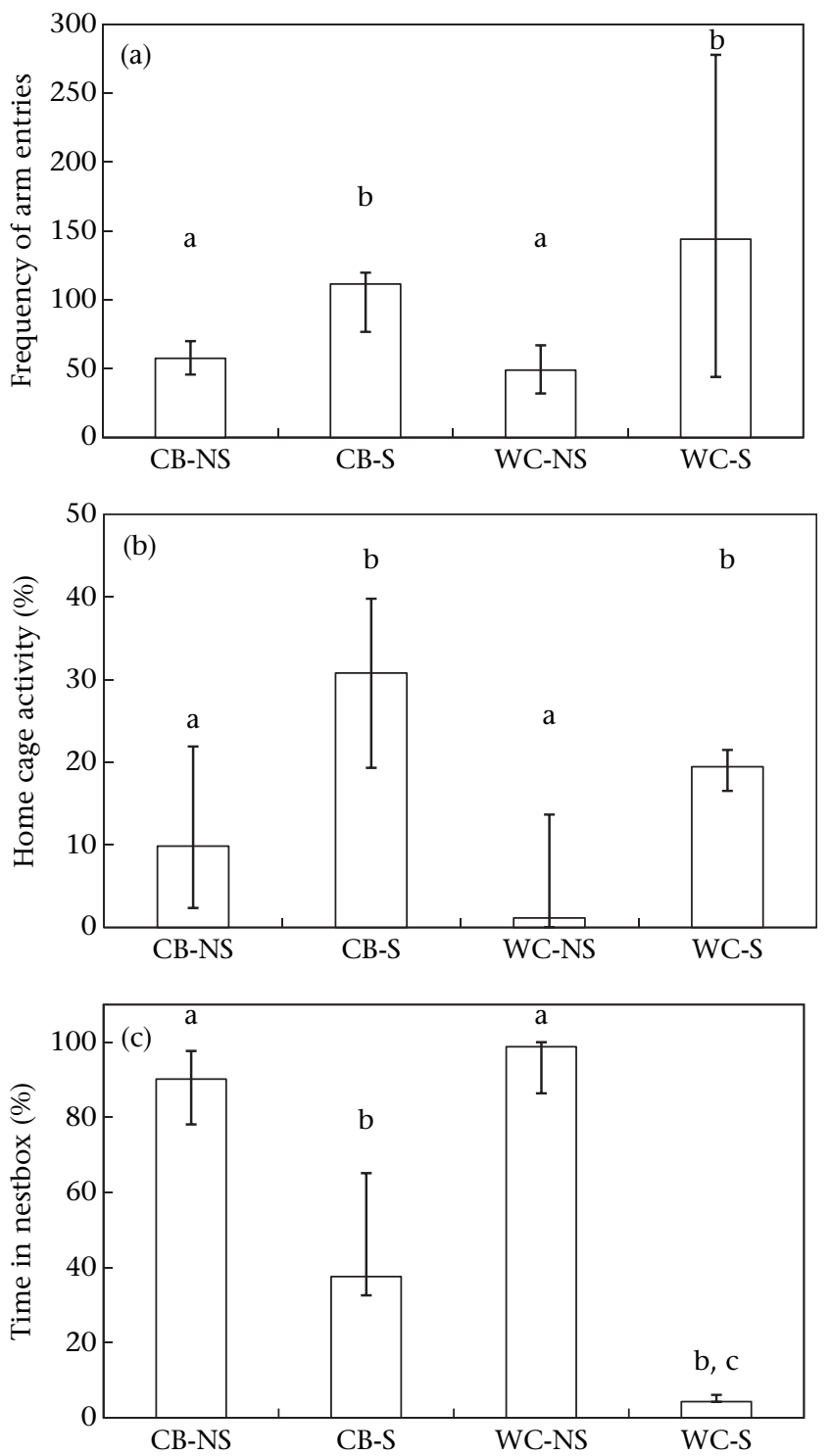

Figure 4. Measures of activity. (a) Frequency of arm entries in the four-arm maze (b) The percentage of active time in the home cage (median, 1st and 3rd quartiles). (c) The percentage of home cage time in the nestbox (median, 1st and 3rd quartiles). $\mathrm{CB}=$ captive-born; $\mathrm{WC}=$ wild-caught; $\mathrm{NS}=$ nonstereotypic; $\mathrm{S}=$ stereotypic. The same alphabet letters denote groups that are not significantly different. Bars = median; whiskers $=1$ st and 3 rd quartiles.

\section{Discussion}

On a group level, the behavioural and physiological profiles of WC and CB randomly selected striped mice differed in several ways. WC striped mice were less perseverative in the four-arm maze, more fearful/anxious in the light-dark box, had higher circulating levels of the unbound (thus active) stress hormone corticosterone, as inferred from faecal metabolites of the hormone, and were less active in both the four-arm maze and in the home cage. Because time hidden in the nestbox during the peak activity period was also significantly predicted by the proportion of time spent in the dark compartment of the light-dark box, we suggest that the extreme inactivity of WC striped mice is a result of fear. When we assessed individual susceptibility to stereotype by accounting for SB status (and using our second data set, which oversampled stereotypic WC mice to permit statistical analysis), birth origin remained a significant predictor of anxiety/fear, but differences between CB and WC striped mice in terms of activity and perseveration disappeared, with these variables better accounted for by the stereotypic status of the animals. Thus the low activity levels and low perseveration of WC animals were statistically associated with their low SB, while their higher stress levels were associated with their birth origin but not stereotypy status. Furthermore, when we assessed the severity of SB from time budget data, similar patterns emerged: striped mice with the most time-consuming SBs were also the most perservative under test (as measured using SA).

Our findings hence support our first prediction that subjects maturing in the wild have greater behavioural flexibility and are less likely to perseverate than $\mathrm{CB}$ conspecifics, and that such effects are indeed related to low/absent SB. Thus, although all stereotypic subjects, irrespective of birth origin, were more inclined to form routines than nonstereotypic subjects, the markedly lower incidence of SB in WC striped mice was accounted for by the much higher proportion of WC than $C B$ striped mice benefitting from enhanced behavioural flexibility. Our results did not, in contrast, support our second prediction that WC striped mice would adjust better to captivity than CB animals. Instead, WC striped mice were generally more anxious/ fearful than their CB counterparts. While it can be argued that anxiety/fearfulness is an adaptive trait under natural conditions, in captivity, where there is no danger of predation, WC animals continually experiencing their environment as threatening (because of their early experience) indicates a problematic animal-environment misfit and can thus be considered a maladjustment. Overall, our results confirm the pattern outlined in the Introduction in which WC animals have poorer welfare than $\mathrm{CB}$ conspecifics. There did not appear to be a direct statistical link between this heightened anxiety/fear and the prevention of SB evident in WC animals (in contrast to prediction 3 ). The elevated SB of those few WC mice that performed this behaviour was an interesting, but unexpected result, and is considered further in the General discussion.

In summary, our results identify specific correlates of the birth origin effect on SB development, which in turn hint at the mechanisms possibly underpinning these birth origin effects on $\mathrm{SB}$, an issue we return to in the General discussion. However, they do not indicate approximately when during development birth origin exerts its enduring influence. Is it sufficient simply to be conceived or gestated in the wild, or do animals additionally need to grow up in the complexity of natural conditions? For example, some studies of environmental enrichment suggest that the brain needs to mature in a complex environment to reap its benefits fully (e.g. Lewis et al. 2006). In experiment 3, we therefore retrospectively analysed historical data to tackle this final question. 


\section{EXPERIMENT 3: AGE AT CAPTURE}

To characterize further the birth origin effects and test whether the effects of being WC on SB were acquired prenatally or postnatally, we retrospectively analysed past laboratory records to investigate effects of age at capture on the development of SB. This approach allowed us to obtain data from a large sample size of WC striped mice without any extra trapping. We hypothesized that age at capture would influence the susceptibility of striped mice to SB development for two interrelated reasons. First, social and physical deprivation early in life is known to affect an organism's brain and behavioural development more adversely than deprivation later in life (Max et al. 2010) and, second, an increased duration of exposure to environmental complexity is thought to confer greater and more lasting protection against later adversity (Nithianantharajah \& Hannan 2006; cf. Lewis et al. 2006). We accordingly predicted that juvenile-caught striped mice would show a higher incidence of SB than adult-caught animals.

\section{Methods}

\section{Study subjects and housing}

We collated and analysed data from WC striped mice trapped and observed in N.P.'s laboratory over the past two decades $(N=204)$, dividing these data into animals trapped as juveniles $(N=103)$ and those trapped as adults $(N=101)$. These striped mice were trapped in four grassland localities in South Africa as part of various socioecological studies between 1993 and 2001: Alice (Eastern Cape; $32^{\circ} 46^{\prime} \mathrm{S}, 2^{\circ} 52^{\prime} \mathrm{E}$ ); Kamberg (KwaZulu-Natal; $29^{\circ} 23^{\prime} \mathrm{S}, 29^{\circ} 42^{\prime} \mathrm{E}$ ); Suikerbosrand (Gauteng; $26^{\circ} 31^{\prime} \mathrm{S}, 28^{\circ} 18^{\prime} \mathrm{E}$ ); and Irene (Gauteng; $25^{\circ} 20^{\prime} \mathrm{S}, 28^{\circ} 9^{\prime} \mathrm{E}$ ). Striped mice used in this study, in accordance with husbandry protocols during this period, were housed in standard Labotec cages (see experiment 1) provisioned with a grass mixture as nesting material.

\section{Procedure}

Immediately after trapping, striped mice were sexed and weighed, and their age was assessed by examining the appearance and/or size of the testes or vaginal opening. Using these parameters, we divided our sample into juvenile-caught mice $(<30 \mathrm{~g}$; nonscrotal/nonperforate) or adult-caught mice ( $>40 \mathrm{~g}$; scrotal/ perforate; De Graaff 1981). Striped mice weighing between 30 and $40 \mathrm{~g}$ were excluded to eliminate individuals whose age class was uncertain (about $10 \%$ of the sample). Four weeks after capture (by which time all juvenile-caught striped mice were adults), individuals were assessed using this laboratory's standard protocol at that time for the absence/presence of SB over a 5-day period (one 15 min observation session per day) as described in experiment 1. Although the WC mice used in this study were not monitored for the development of SB after this observation phase, we have only once incidentally observed a WC striped mouse developing SB later than 4 weeks after capture (unpublished data; also see experiment 1 ), and so this protocol should classify age class accurately.

\section{Data analysis}

We analysed these categorical data using logistic regression with a binomial logit function, including sex, trapping locality, age class and sex*age class as categorical predictors, and SB status as the dependent variable.

\section{Results}

There were no overall effects of sex (Wald $\chi_{1}^{2}=0.37, P=0.54$ ) or trapping locality (Wald $\chi_{1}^{2}=3.95, P=0.27$ ) on SB. However, age at capture significantly predicted the development of SB (Wald

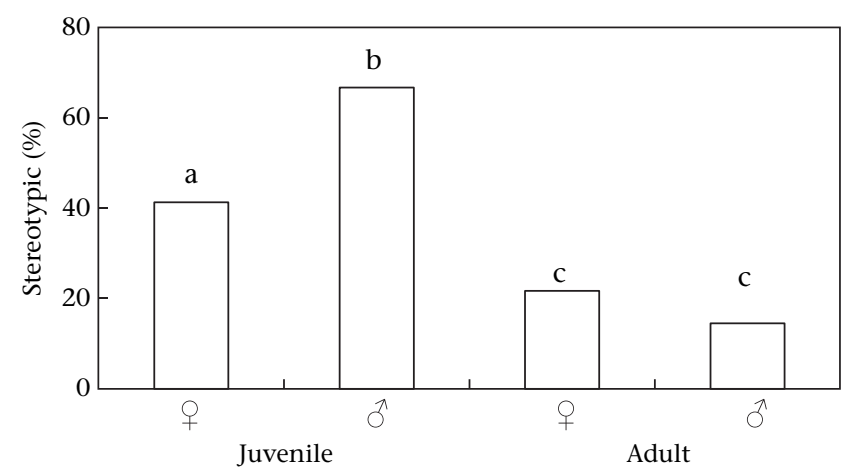

Figure 5. The percentage of juvenile and adult female and male WC striped mice that developed stereotypic behaviour after 4 weeks of captive housing.

$\left.\chi_{1}^{2}=26.51, P<0.001\right): 55 \%$ of juveniles developed SB compared with only $19 \%$ of adults, but there was a significant age*sex interaction (Wald $\chi_{1}^{2}=5.71, P=0.017$ ) because males were more affected by age at capture than females (Fig. 5). When we reanalysed the data separately for males and females, age class at capture remained a significant predictor of SB development in both sexes (males: Wald $\chi_{1}^{2}=25.58, P<0.001$; females: Wald $\chi_{1}^{2}=4.28$, $P=0.039)$.

\section{Discussion}

As predicted, birth origin effects were mediated by age at capture: the incidence of SB was significantly lower in adult-caught striped mice than juvenile-caught individuals (19\% versus 55\%), with juvenile-caught striped mice displaying an incidence of SB similar to that of CB individuals ( $55 \%$ versus $57 \%$ ). It appears that an extended period of early environmental complexity is thus necessary to protect striped mice against SB development, with the beneficial effects of birth origin accruing predominantly postnatally. Unexpectedly, male juvenile-caught striped mice were more severely affected by early removal from the wild than female juvenile-caught individuals, possibly reflecting a differential impact of captivity on juvenile males and females.

\section{GENERAL DISCUSSION}

As in most other species of wild animals studied to date (see Introduction), our findings showed that WC striped mice were less likely to develop SB than CB conspecifics. Unique to this study, however, was our use of CB and WC striped mice that had been kept in the same laboratory which allowed us to investigate the associated correlates of birth origin on SB, with potential implications for all captive wild species kept and bred in laboratories, zoos and breeding centres. The better understanding of these birth origin effects is important for a number of reasons. First, SB and its correlates reflect profound alterations in behaviour, and perhaps even indicate atypical CNS development in CB wild animals compared to their WC counterparts. This has implications for the validity of ethological studies using $\mathrm{CB}$ animals to model behavioural processes in WC animals and, moreover, could impact on the success (or otherwise) of captive-breeding and release programmes. Second, although the absence of SB typically indicates conditions associated with good welfare (e.g. Mason \& Latham 2004), the low levels of SB seen in WC animals seem to represent an important exception to this general 'low SB-good welfare' link.

Our analyses showed that striped mice had to reach adulthood in the wild to achieve maximal protection against SB development. Here, sex played an interactive role, with juvenile males four times 
as likely to develop SB as WC adult males, while females were only twice as likely to become stereotypic if caught as juveniles rather than as adults. In consequence, juvenile-caught males were more stereotypic than both juvenile-caught females and wild-caught adults of either sex, and were even as prone to becoming stereotypic as $\mathrm{CB}$ animals. These findings suggest that both age at capture and sex may be important determinants of the behavioural phenotype of WC animals in laboratories, zoos and conservation breeding centres. Furthermore, since all the striped mice, even juvenile-caught, were captured post weaning age (after which time grassland striped mice are solitary; Schradin \& Pillay 2005), this suggests that the physical aspects of environmental space and complexity, and/or an extended period of autonomous decision making, may underlie the effects of birth origin and not, for instance, that of the premature loss of parental care (a common confound in other CB-WC comparisons; Mason 2006; Latham \& Mason 2008).

Instead of being stereotypic, WC striped mice were extremely inactive within their home cages, spending most of their time in their nestboxes when they otherwise would be expected to be active. Similar high levels of inactivity or being out of sight within an enclosure are commonly reported in many species kept in zoos (e.g. Meyer-Holzapfel 1968; Altman 1999), and our findings now suggest this may be a particular problem in WC animals. Because WC mice were also more stressed and fearful, and because there was a significant association between time in the nestbox and separate behavioural measures of fear, these results also suggest that this extreme inactivity may well be a manifestation of poor welfare In addition, the few WC striped mice to develop SBs showed two notable characteristics. First, they developed only one form of SB, circuit running, while CB animals displayed a greater variety of SBs. Second, the stereotypic WC striped mice devoted significantly more time to SB than their stereotypic CB peers. Thus, when WC striped mice stereotyped, the SBs appeared to be more severe and perhaps more frenetic, a phenomenon now needing investigating in other captive wild species. The exclusive use of circuit running by WC striped mice was unexpected, but could reflect a greater a priori physical fitness than in CB animals and/or frustrated ranging; free-ranging grassland striped mice have home ranges of about $1000 \mathrm{~m}^{2}$ (Schradin \& Pillay 2005), some 16000 times bigger than the laboratory cages, and frustrated ranging opportunities have been shown to underpin locomotor stereotypies in the Carnivora (Clubb \& Mason 2003, 2007). In passerine bird species, WC individuals displayed a similar preference for routetracing SBs over other forms of SB such as 'spot picking' (Keiper 1969).

Our investigations of perseveration, fear/anxiety and activity revealed substantial effects of birth origin, with some, but not all variables correlating with SB and so reflecting potential mediators of the link between birth origin and of SB development. The clearest correlate of SB that might account for the effect of birth origin was perseveration. Thus on a group level, WC striped mice showed more flexible patterns of arm entry in the four-arm maze but, when we controlled for SB, the effect of birth origin disappeared: stereotypic striped mice, regardless of birth origin, were more perseverative than their nonstereotypic counterparts. Scores on one of our measures of perseveration, SA, also predicted the duration of time engaged in SB. These behavioural results thus suggest that, like deprivation-reared primates, and like deer mice developing in standard versus enriched housing conditions (Lewis et al. 2006; Latham \& Mason 2008), forebrain development is altered in $\mathrm{CB}$ animals in a manner that decreases both behavioural flexibility and the ability to inhibit inappropriate behavioural repetitions, with perseveration and SB being two outcomes of such a change. A second factor associated with SB was activity.
Stereotypic striped mice were, as discussed, more active than nonstereotypic individuals, an observation agreeing with findings in other animals (e.g. bank voles) in which enhanced rates of behavioural initiation are thought to predispose both hyperactivity and SB (Garner \& Mason 2002).

The regional activity of specific forebrain areas (candidates include the motor cortex, striatum, nucleus accumbens and thalamus) should now be identified using such techniques as pharmacological probes (e.g. Schoenecker \& Heller 2000; Presti et al. 2003) or post mortem histology, for instance for dendritic branching or metabolic activity (via cytochrome oxidase staining; e.g. McBride \& Hemmings 2005; Lewis et al. 2006). Our results suggest altered striatal function in CB mice compared to WC mice; they predict changes in such regions that predate the emergence of SB and also predict correlational associations between the magnitude of such effects and perseveration and SB scores. Such studies might similarly help explain the way the age-at-capture effect interacted with sex, since the trajectories of brain maturation in human children and adolescents are influenced by sex, with maltreatment-induced abnormalities in brain development more common in males, possibly because of hormonally driven differences in dendritic arborization, pruning and myelination (De Bellis et al. 2001). One remaining question here, however, is whether the better predictor of SB development is (1) the effect of age at capture (and the developmental stage of the CNS), (2) the duration of time spent in the wild or (3) both of these. Additional further studies would be necessary to tease apart these confounding variables and thus the effects of sex, developmental stage, age and early environmental risk factors on the predisposition to stereotype.

Despite SB usually indicating group-level poor welfare (see Introduction), the fact that WC striped mice were less stereotypic than $\mathrm{CB}$ animals did not necessarily mean that they had better welfare. In fact, as summarized above, WC individuals (irrespective of SB status) were more anxious/fearful than CB striped mice in behavioural tests, and they had higher circulating levels of corticosterone. Thus, the virtual absence of SB in WC animals might be an important exception to the 'low SB-good welfare' rule of thumb. The very low activity levels of WC striped mice seemed to be an alternative behavioural response to adverse captive conditions. Future work should investigate more fully whether this inactivity reflects (1) fear-mediated hiding in the nestbox (for instance using tests in which fear cannot be confounded with activity, or nestboxes that vary in the degree of cover they offer) or (2) depressionrelated 'apathy', a hypothesis that could be assessed by running tests for anhedonia (e.g. Willner et al. 1996) in CB versus WC animals and investigating its links with home cage inactivity. A third sign of reduced welfare in WC animals is that the few WC striped mice that did stereotype did so at significantly higher levels than seen in CB mice. This suggests that they may be more frustrated by the confines of captivity than animals born into it and that, for those few individuals susceptible to SB development because of their perseverative tendencies, this frustration exacerbates the performance of SBs. Any subsequent work should therefore also test directly for differences in motivational frustration between WC and CB individuals (as has been done between previously enriched laboratory mice and their standard-housed counterparts; Latham \& Mason 2010), and for the relationships between SB and frustration within these two groups.

In conclusion, our results show that WC striped mice, provided they are trapped as adults, are typically protected from the development of SB when captive housed and are also less perseverative under test, suggesting intact forebrain function. WC striped mice (whether stereotypic or not) none the less adjust more poorly to captivity than those animals born into it. These results suggest that 
striped mice are a promising species in which to investigate the neurological mechanisms underpinning the pervasive effects of birth origin, and indicate the need for further investigations in other species to test, for instance, whether and how birth origin and age at capture, inactivity, welfare and captive conditions interact to shape phenotype. Finally, given the likely role of physical environmental complexity in mediating behavioural flexibility via influencing forebrain development, it may well also be worth considering on ethical grounds whether enriched-reared CB striped mice would be better suited subjects for such experiments, thus minimizing the need for future trapping and use of highly anxious/ fearful WC animals.

\section{Acknowledgments}

Funding was provided by the National Research Foundation (NRF; grant number: 2069110) and the University of the Witwatersrand to N.P. M.J. was partly supported during her Ph.D. by an NRF Scarce Skills Scholarship and, for this work, by a grant from the Universities Federation of Animal Welfare (UFAW). G.M. was supported by an NSERC Canada Research Chair and Discovery Grant. M.J. thanks Tasmin Rymer, Sneha Joshi and Alessia Fowler for help with data collection, Rebecca Meagher for her insightful comments and assistance with references, Jamie Dallaire for his generous after-hours' discussion of data, Lis Jones for skilfully proofreading the manuscript and two anonymous referees for their helpful comments.

\section{References}

Altman, J. D. 1999. Effects of inedible, manipulable objects on captive bears. Journal of Applied Animal Welfare Science, 2, 123-132.

Belzung, C. \& Griebel, G. 2001. Measuring normal and pathological anxiety-like behaviour in mice: a review. Behavioural Brain Research, 125, 141-149.

Biggins, D. E., Vargas, A., Godbey, J. \& Anderson, S. H. 1999. Influence of prerelease experience on reintroduced black-footed ferrets (Mustela nigripes). Biological Conservation, 89, 121-129.

Bourin, M. \& Hascoët, M. 2003. The mouse light/dark box test. European Journal of Pharmacology, 463, 55-65.

Broom, D. M. \& Johnson, K. G. 1993. Stress and Animal Welfare. Dordrecht: Kluwer/ Chapman \& Hall.

Carlstead, K., Mellen, J. \& Kleiman, D. G. 1999. Black rhinoceros (Diceros bicornis) in U.S. zoos: I. Individual behavior profiles and their relationship to breeding success. Zoo Biology, 18, 17-34.

Clubb, R. \& Mason, G. 2003. Captivity effects on wide-ranging carnivores. Nature, 425, 473.

Clubb, R. \& Mason, G. J. 2007. Natural behavioural biology as a risk factor in carnivore welfare: how analysing species differences could help zoos improve enclosures. Applied Animal Behaviour Science, 102, 303-328.

Clubb, R., Lee, P., Mar, K. U., Moss, C., Rowcliffe, M. \& Mason, G. J. 2008. Compromised survivorship in zoo elephants. Science, 322, 1649.

Delatour, B. \& Gisquet-Verrier, P. 1996. Prelimbic cortex specific lesions disrupt delayed-variable response tasks in the rat. Behavioral Neuroscience, 110, 1282-1298.

De Bellis, M. D., Keshavan, M. S., Beers, S. R., Hall, J., Frustaci, K., Maselehdan, A., Noll, J. \& Boring, A. M. 2001. Sex differences in brain maturation during childhood and adolescence. Cerebral Cortex, 11, 552-557.

De Graaff, G. 1981. The Rodents of Southern Africa: Notes on their Identification, Distribution, Ecology and Taxonomy. Durban: Butterworths.

Divac, I., Wikmark, R. G. E. \& Gade, A. 1975. Spontaneous alternation in rats with lesions in the frontal lobes: an extension of the frontal lobe syndrome. Physiological Psychology, 3, 39-42.

Feenders, G. \& Bateson, M. 2011. Hand-rearing reduces fear of humans in European starlings, Sturnus vulgaris. PLoS ONE, 6, e17466.

Garner, J. P. 2006. Perseveration and stereotypy: systems-level insights from clinical psychology. In: Stereotypic Animal Behaviour: Fundamentals and Applications to Welfare (Ed. by G. Mason \& J. Rushen), pp. 121-152. 2nd edn. Oxford: САВ International.

Garner, J. P. \& Mason, G. J. 2002. Evidence for a relationship between cage stereotypies and behavioural disinhibition in laboratory rodents. Behavioural Brain Research, 136, 83-92.

Garner, J. P., Mason, G. J. \& Smith, R. 2003. Stereotypic route-tracing in experimentally caged songbirds correlates with general behavioural disinhibition. Animal Behaviour, 66, 711-727.

Graybiel, A. M. 2008. Habits, rituals and the evaluative brain. Annual Review of Neuroscience, 31, 359-387.
Ha, J. C., Robinette, R. L. \& Davis, A. 2000. Survival and reproduction in the first two years following a large-scale primate colony move and social reorganization. American Journal of Primatology, 50, 131-138.

Hlinák, Z. \& Krejči, I. 2006. Spontaneous alternation behaviour in rats: kynurenic acid attenuated deficits induced by MK-801. Behavioural Brain Research, 168, 144-149.

Imaizumi, M., Miyazaki, S. \& Onodera, K. 1994. Effects of xanthine derivatives in a light/dark test in mice and contribution of adenosine receptors. Methods and Findings in Experimental and Clinical Pharmacology, 16, 639-644.

Joel, D., Stein, D. J. \& Schreiber, R. 2008. Animal models of obsessive-compulsive disorder: from bench to bedside via endophenotypes and biomarkers. In: Animal and Translational Models for CNS Drug Discovery. Vol. 1 of 3: Psychiatric Disorders (Ed. by R. McArthur \& F. Borsini), pp. 134-154. London: Academic Press.

Jones, M., van Lierop, M. \& Pillay, N. 2008. All a mother's fault? Transmission of stereotypy in striped mice Rhabdomys. Applied Animal Behaviour Science, 115, 82-89.

Jones, M. A., Mason, G. \& Pillay, N. 2010a. Early social experience influences the development of stereotypic behaviour in captive-born striped mice Rhabdomys. Applied Animal Behaviour Science, 123, 70-75.

Jones, M. A., van Lierop, M., Mason, G. \& Pillay, N. 2010b. Increased reproductive output in stereotypic captive Rhabdomys females: potential implications for captive breeding. Applied Animal Behaviour Science, 123, 63-69.

Keiper, R. R. 1969. Causal factors of stereotypies in caged birds. Animal Behaviour, 17, $114-119$.

Kim, S., Lee, S., Ryu, S., Suk, J. \& Park, C. 2002. Comparative analysis of the anxietyrelated behaviors in four inbred mice. Behavioural Processes, 60, 181-190.

Kleiman, D., Beck, B., Baker, A., Ballou, J., Dietz, L. \& Dietz, J. 1990. The conservation program for the golden lion tamarin, Leontopithecus rosalia. Endangered Species Update, 8, 82-85.

Lalonde, R. 2002. The neurological basis of spontaneous alternation. Neuroscience and Biobehavioral Reviews, 26, 91-104.

Latham, N. R. \& Mason, G. J. 2008. Maternal deprivation and the development of stereotypic behaviour. Applied Animal Behaviour Science, 110, 84-108.

Latham, N. \& Mason, G. 2010. Frustration and perseveration in stereotypic captive animals: is a taste of enrichment worse than none at all? Behavioural Brain Research, 211, 96-104.

Lewis, M. H., Presti, M. F., Lewis, J. B. \& Turner, C. A. 2006. The neurobiology of stereotypy I. environmental complexity. In: Stereotypic Anima Behaviour: Fundamentals and Applications to Welfare (Ed. by G. Mason \& J. Rushen), pp. 190-226. 2nd edn. Oxford: CAB International.

McBride, S. D. \& Hemmings, A. 2005. Altered mesoaccumbens and nigro-striatal dopamine physiology is associated with stereotypy development in a nonrodent species. Behavioural Brain Research, 159, 113-118.

Mason, G. 2006. Are wild-born animals 'protected' from stereotypies? In: Stereotypies in Captive Animals (Ed. by G. Mason \& J. Rushen), p. 196. 2nd edn. Wallingford: CAB International.

Mason, G. J. \& Latham, N. R. 2004. Can't stop, won't stop: is stereotypy a reliable animal welfare indicator? Animal Welfare, 13, S57-S69.

Mason, G., Clubb, R., Latham, N. \& Vickery, S. 2007. Why and how should we use environmental enrichment to tackle stereotypic behaviour? Applied Animal Behaviour Science, 102, 163-188.

Marin, R. S. 1990. Differential diagnosis and classification of apathy. American Journal of Psychiatry, 147, 22-30.

Mathews, F., Orros, M., McLaren, G., Gelling, M. \& Foster, R. 2005. Keeping fit on the ark: assessing the suitability of captive-bred animals for release. Biological Conservation, 121, 569-577.

Max, J. E., Bruce, M., Keatley, E. \& Delis, D. 2010. Pediatric stroke: plasticity vulnerability, and age of lesion onset. Journal of Neuropsychiatry and Clinical Neurosciences, 22, 30-39.

Meyer-Holzapfel, M. 1968. Abnormal behavior in zoo animals. In: Abnormal Behavior in Animals (Ed. by M. W. Fox), pp. 476-503. Philadelphia: W. B. Saunders.

Nel, K. N. 2003. The effects of dietary protein on the reproduction and behavioural characteristics of the striped mouse, Rhabdomys pumilio. M.Sc. thesis, University of the Witwatersrand.

Nevison, C. M., Hurst, J. L. \& Barnard, C. J. 1999. Why do male ICR(CD-1) mice perform bar-related (stereotypic) behaviour? Behavioural Processes, 47, 95-111.

Nithianantharajah, J. \& Hannan, A. J. 2006. Enriched environments, experiencedependent plasticity and disorders of the nervous system. Nature Reviews Neuroscience, 7, 697-709.

Nogueira, S. S. C., Bernardi, L. G. \& Nogueira-Filho, S. L. G. 2004. A note on comparative enclosure facility usage by wild and captive-born capybaras (Hydrochoerus hydrochaeris). Applied Animal Behaviour Science, 89, 139-143.

Perry, J. M., Izard, M. K. \& Fail, P. A. 1992. Observations on reproduction, hormones, copulatory behaviour, and neonatal mortality in captive Lemur mongoz (Mongoose lemur). Zoo Biology, 11, 81-87.

Pillay, N. 2000. Female mate preference and reproductive isolation in populations of the striped mouse Rhabdomys pumilio. Behaviour, 137, 1431-1441.

Presti, M. F., Mikes, H. M. \& Lewis, M. H. 2003. Selective blockade of spontaneous motor stereotypy via intrastriatal pharmacological manipulation. Pharmacology, Biochemistry, and Behavior, 74, 833-839.

Robbins, T., Mittleman, G., O'Brien, J. \& Winn, P. 1990. The neurobiological significance of stereotypy induced by stimulant drugs. In: The Neurobiology of Stereotyped Behaviour (Ed. by S. Cooper \& C. Dourish), pp. 25-63. Oxford: Clarendon Press.

Sandson, J. \& Albert, M. 1987. Perseveration in behavioural neurology. Neurology, 37, 1736-1741. 
Schoenecker, B. \& Heller, K. E. 2000. Indication of a genetic basis of stereotypies in laboratory-bred bank voles (Clethrionomys glareolus). Applied Animal Behaviour Science, 68, 339-347.

Schradin, C. 2006. Whole-day follows of striped mice (Rhabdomys pumilio), a diurnal murid rodent. Journal of Ethology, 24, 37-43.

Schradin, C. \& Pillay, N. 2003. Paternal care in the social and diurnal striped mouse (Rhabdomys pumilio): laboratory and field evidence. Journal of Comparative Psychology, 117, 317-324.

Schradin, C. \& Pillay, N. 2005. Intraspecific variation in the spatial and socia organization of the African striped mouse. Journal of Mammalogy, 86, 99-107.

Schwaibold, U. \& Pillay, N. 2001. Stereotypic behaviour is genetically transmitted in the African striped mouse Rhabdomys pumilio. Applied Animal Behaviour Science, 74, 273-280.

Skinner, J. D. \& Chimimba, C. T. 2005. The Mammals of the Southern African Subregion. 3rd edn. Cape Town: Cambridge University Press.

Swaisgood, R. R., Dickman, D. M. \& White, A. M. 2006. A captive population in crisis: testing hypotheses for reproductive failure in captive-born southern white rhinoceros females. Biological Conservation, 129, 468-476.

Tanimura, Y., Yang, M. G. \& Lewis, M. H. 2008. Procedural learning and cognitive flexibility in a mouse model of restricted, repetitive behaviour. Behavioural Brain Research, 189, 250-256.

Touma, C. \& Palme, R. 2005. Measuring fecal glucocorticoid metabolites in mammals and birds: the importance of validation. Annals of the New York Academy of Sciences, 1046, 54-74.
Touma, C., Sachser, N., Mostl, E. \& Palme, R. 2003. Effects of sex and time of day on metabolism and excretion of corticosterone in urine and feces of mice. General and Comparative Endocrinology, 130, 267-278.

Touma, C., Palme, R. \& Sachser, N. 2004. Analyzing corticosterone metabolites in fecal samples of mice: a noninvasive technique to monitor stress hormones. Hormones and Behavior, 45, 10-22.

Ulloa, R., Nicolini, H. \& Fernández-Guasti, A. 2004. Sex differences on spontaneous alternation in prepubertal rats: implications for an animal model of obsessive-compulsive disorder. Progress in Neuro-Psychopharmacology $\mathcal{E}$ Biological Psychiatry, 28, 687-692.

Van Hooff, J. A. R. A. M. 1982. Categories and sequences of behavior: methods of description and analysis. In: Handbook of Non-Verbal Communication Research (Ed. by P. Ekman \& K. Scherer), pp. 362-439. New York: Cambridge University Press.

Vickery, S. S. \& Mason, G. J. 2003. Behavioral persistence in captive bears: implications for reintroduction. Ursus, 14, 35-43.

Wiedenmayer, C. 1997. Causation of the ontogenetic development of stereotypic digging in gerbils. Animal Behaviour, 53, 461-470.

Willner, P., Moreau, J., Nielsen, C. K., Papp, M. \& Sluzewska, A. 1996. Decreased hedonic responsiveness following chronic mild stress is not secondary to loss of body weight. Physiology \& Behavior, 69, 129-134.

Yadin, E., Friedman, E. \& Bridger, W. H. 1991. Spontaneous alternation behavior: an animal model for obsessive-compulsive disorder. Pharmacology Biochemistry E' Behavior, 40, 311-315. 